\title{
ATLAS-BASED SEGMENTATION OF MEDICAL IMAGES LOCALLY CONSTRAINED BY LEVEL SETS
}

\author{
Valérie Duay, Nawal Houhou and Jean-Philippe Thiran \\ Signal Processing Institute (ITS), \\ Ecole Polytechnique Fédérale de Lausanne (EPFL) \\ CH-1015 Lausanne, Switzerland \\ \{Valerie.Duay, Nawal.Houhou, JP.Thiran\}@epfl.ch
}

\begin{abstract}
Atlas-based segmentation has become a standard paradigm for exploiting prior knowledge in medical image segmentation. In this paper, we propose a method to exploit both the robustness of global registration techniques and the accuracy of a local registration based on level set tracking. First, the atlas is globally put in correspondence with the patient image by an affine and an intensity-based non rigid registration. Based on this rough initialisation, the level set functions corresponding to particular objects of interest of the deformed atlas are used to segment the corresponding objects in the patient image. We propose a technique to derive a dense deformation field from the motion of these level set functions. This is particularly important when we want to infer the position of invisible structures like the brain sub-thalamic nuclei from the position of visible surrounding structures. This can also be advantageously exploited to register an atlas following a hierarchical approach. Results are shown on 2D synthetic images and 2D real images extracted from brain and prostate MR volumes and neck CT volumes.
\end{abstract}

\section{INTRODUCTION}

Atlas-based segmentation of medical images has become a standard paradigm for exploiting prior anatomical knowledge in image segmentation. Some of the most critical requirements of atlasbased segmentation, particularly in radiation therapy or neurosurgical planning, are the following: the contours of segmented structures have to be found as accurately as possible, while staying well smooth, the connectivity relationships between structures defined in the atlas have to be maintained through the registration / segmentation process and the segmentation of structures without visible edge, i.e. contours only defined with respect to adjacent structures, has to be possible. In the majority of the approaches proposed so far to register an atlas to a patient image, the objective of the transformation is to optimize some global intensity-based correspondence measure (like level-gray differences, regional correlation, or mutual information). Some recent algorithms like [1] combine global and more local intensity-based registration. This permits to improve the results while decreasing the computation time. However this is not yet sufficient to guaranty the desired quality of segmentation for the most demanding applications. Most of the time the only constraint used on the transformation is its smoothness, ensured for instance by a Gaussian filtering [2] or constraints between interpolation functions [3]. When at some places contours are not accurate enough, it is usual to globally or locally allow more elasticity to the deformation in order to obtain a more local deformation, with the risk of increasing the irregularity of the deformation field and thus of the contours, without necessarily obtaining the sought level of precision.

To cope with this problem, additional constraints have to be included in these types of intensity-based registration algorithms. These constraints should permit to impose the smoothness of the contours while introducing more local a priori information such the intensity distribution or the admissible shapes of objects to be segmented. The level set theory is particularly well appropriated to define and implement such constraints.

Methods have already been proposed to use level set techniques into an atlas registration process. In [4], D'Haese et al. perform a post-processing via level set after an intensity-based parametric registration to improve the final segmentation. Baillard et al. [5] initialize level set surfaces close to the patient contours by an optical flow registration before finalizing the segmentation with level sets. Two groups, Vermuri et al. [6] and Bertalmio et al. [7] reformulated the registration problem into a curve evolution approach and implemented it into a level set framework. The main difference between the Vemuri and Bertalmio methods is that Bertalmio registration governing equation involves not only one partial differential equation (PDE) for morphing the image intensities as in the Vermuri formulation but also another one coupled with the first one for morphing the image contours.

In this paper, we present a level set-based local registration method permitting to bring more local prior information in the atlas registration. Atlas registration process is usually constituted of two steps: a rigid or affine registration following by a non rigid registration. Our method is compatible with this framework, but adds a third step.

\section{METHOD}

The atlas-based segmentation relies on the existence of a reference image (the atlas) in which objects of interest have been carefully segmented, manually or with the help of a semi-automatic segmentation method. We consider that binary masks have been created for each object from these segmentations. To segment a new image (a patient), a transformation that registers (i.e puts in point-topoint spatial correspondence) the atlas to the patient image has to be computed. This transformation will be then used to deform the binary masks from the atlas onto the patient image to segment it. The process that we propose to establish this point-to-point correspondence is illustrated in Figure 1. 


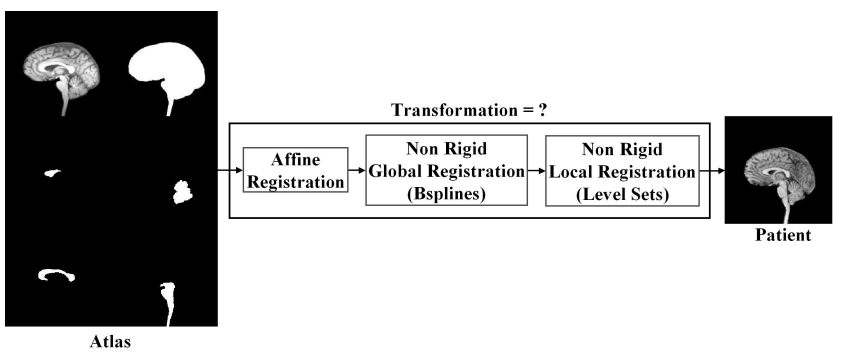

Fig. 1. Atlas-based segmentation process.

The novelty is that this registration is performed in three steps instead of two. Each of these steps allows more and more degrees of freedom to the registration in order to compute the transformation, from global to local. This permits to reduce the risk to fall in local minima during the registration while speeding up the registration process. These three steps are described in more details below.

\subsection{Affine registration}

First, the atlas is registered to the patient image with an affine transformation (twelve degrees of freedom) using mutual information and various resolution levels.

\subsection{Global non rigid registration}

Then an intensity-based algorithm performs a global non rigid registration. For this study, we have chosen to use a parametric algorithm. In this technique, inspired by the work of Rueckert et al. [8], the deformation that registers the affine registration result to the patient is modelled by a linear combination of basis functions:

$$
x^{\prime}=x+\sum_{i=1, \ldots, N} c_{i} \phi_{i}\left(x_{i}-x\right)
$$

where $x$ is a spatial position in one image, $x^{\prime}$ is the corresponding point in the other image, $\phi_{i}$ are the basis functions and $c_{i}$ are the coefficients.

In our implementation, we use Bsplines placed on a regular grid. An evolution algorithm is used to optimize the basis function coefficients following the mutual information between both images. The algorithm computes the final deformation field iteratively across scales and resolutions. In this context, resolution means the spatial resolution of the image while the scale is related to the number of basis functions used, the more basis functions, the more degrees of freedom allowed to the transformation. To keep the transformation smooth, we filter the coefficients of adjacent basis functions with a Gaussian kernel during their optimization. Through this multi-scale multi-resolution approach, it is possible to achieve good registration results, and to compensate large differences in images, like the position of the spinal cord in MR images of the head and neck. This type of algorithm however requires a lot of tests with different number of scales and resolutions to obtain the desired quality of registration. With the level set-based local registration that we propose here, it is not anymore necessary to reach this optimal solution. Just a good approximation of the final registration is sufficient. The accuracy of the registration will be achieved at the third step of the process.

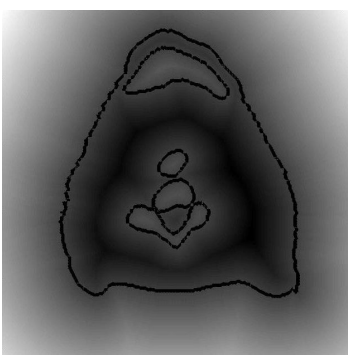

(a)

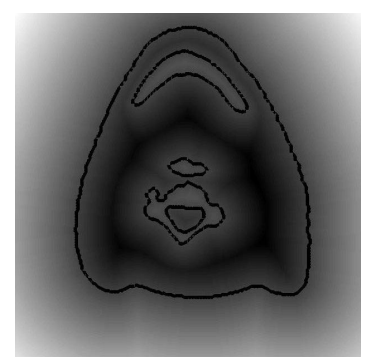

(b)
Fig. 2. Level set tracking.

\subsection{Local non rigid registration}

Our local non rigid registration algorithm permits to benefit from the advantages of the level set techniques in order to obtain a more accurate registration on the contours of the interest objects.

\section{A. Level set evolution equation}

To register the structures of the atlas with the patient image, the level set function corresponding to each object of interest is first computed. A level set function is in fact a signed distance map computed from the contours (positive inside, negative outside and null on the contours). The original shapes of these level sets are given by the atlas binary masks deformed by the current transformation (i.e. the combination of the transformation computed during the first and second step of the registration process). Then each level set evolves independently following a partial differential equation. The PDE used in our implementation contains four terms: a term attracting the active contour to the contours of the patient image, a term setting its speed of propagation, another term limiting its curvature and a region-based term working as a competition between the entropy of the region to segment and the entropy of the background region. At each of these terms a scalar constant is associated, permitting to weight their relative influence on the movement of the level set function. The three first terms are those described in the paper of Caselles et al. [9] and are available in the itk level set library [10]. The equation of the region term has been proposed by A. Herbulot et al. in [11].

\section{B. Extraction of the deformation field}

To obtain an explicit deformation field from the level set motions, two new level sets are computed. One from the original contours of the object of interest and another one from these same contours after the evolution of their level set on the patient image. Figure 2 shows these two level sets for the local registration of two neck slices. The contours of figure 2(a) are those obtained after the Bsplines registration.

Each point of these level set functions is then tracked with the following gradient-based equation:

$$
\overrightarrow{D F}(x)=(\psi(x, \tau)-\psi(x, \tau-1))\|\nabla \psi(x, \tau-1)\|
$$

where $\psi(x, \tau)$ is the level set function at time $\tau$ and $\overrightarrow{D F}(x)$ is the motion vector of the pixel $x$ between the instant $\tau-1$ and $\tau$. $\|\nabla \psi(x, \tau-1)\|$ has to be filtered previously by a gradient vector flow (GVF). This is to address problems of poor convergence associated with boundary concavities. GVF is computed as a diffusion 
of the gradient vectors $\|\nabla \psi(x, \tau-1)\|$ (for more details about this filter, see [12]).

The obtained deformation field permits to propagate the motion of the level set functions to the whole atlas image.

\section{The hierarchical registration}

In addition to this global and local registration, we also propose here to perform the registration in a hierarchical way. In this approach, the more visible and important objects are registered first. Thus, the first layer of the hierarchy contains a subset of the atlas with its most important contours. The resulting deformation field is then used as initial condition for the registration of the next layer including less visible and more complex objects. The goal is that the registration of the objects of one hierarchical layer helps the segmentation of the objects of the next ones. While the contours of the objects concerned by the current layer are registered, the atlas contours of the objects defined in the next layers of the hierarchy are brought closer to their target contours in the patient image, thanks to the level set tracking algorithm presented here above.

\section{RESULTS}

\subsection{Synthetic Images}

The performance of our local atlas-based registration algorithm was first evaluated on 2D binary images of size $256 \times 256$. The source image contains a pentagon and the target image a star. The two shapes are aligned on the same center. The test consists in deforming the pentagon into the star only using the level set-based registration. Note that these two shapes were designed to permit the evolution of the level set in the inside and outside direction (expansion and contraction). Figure 3 presents the obtained results. Panel 3(a) shows original differences between the source and the target image (common sections are showed in white, regions that do not correspond are shown in gray). Panel 3(b) shows the differences after registration of the source image. Panel 3(c) shows a test image that has the same shape as the source image but that contains concentric lines with different intensity values to help in visualizing the transformation. Panel 3(d) shows the shape on the panel 3(c) deformed with the computed transformation and the panel 3(e) shows the transformation applied to a regular grid and added on the deformed source image. Panels 3(d) and 3(e) show that the deformation field extract from the level set motion tracking presents the advantages to be well regular and perpendicular to the contours. Notice that the central area of the pentagon in panel 3(c) has practically disappeared in panel 3(d). This is due to the propagation of the contraction movement of the pentagon to form the concave part between two star points.

\subsection{Medical Images}

We have tested our algorithm on three different cases on 2D medical images of size 256x256. These images were generated by extracting two similar slices of two different patient volumes after affine registration.

The first experience (Figure 4) consists in registering two CT slices of the neck region using four level sets corresponding to the external neck contours, the vertebra, the trachea and the jaw. In this case, the hierarchical approach was not used. To show the

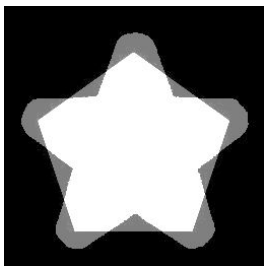

(a)

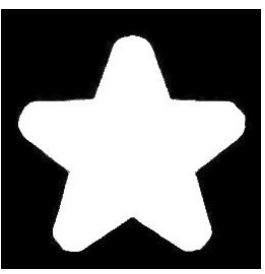

(b)

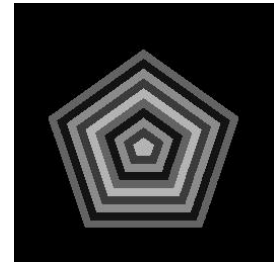

(c)

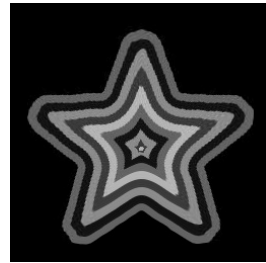

(d)

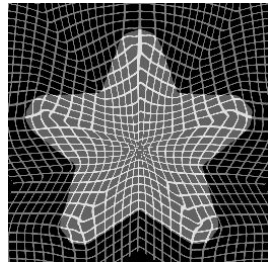

(e)
Fig. 3. Level set-based registration of binary images.

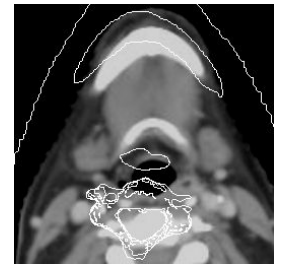

(a)

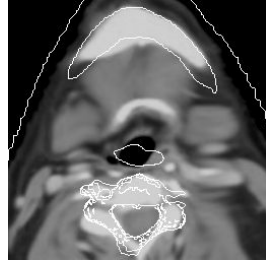

(b)

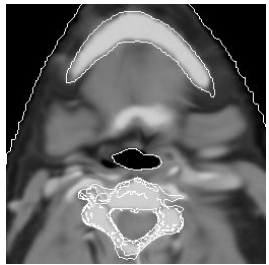

(c)
Fig. 4. Atlas-based registration of a neck axial slice.

accuracy of the registration during the process, contours of interest objects have been drawn on the patient image and copied on all the other panels. Panel 4(a) shows the atlas image after the affine registration. Panel 4(b) shows the result after the global non rigid registration. Panel 4(c) shows the final result after the level setbased registration.

The second experience (Figure 5) consists in registering brain sagittal slices. In this case, the hierarchical approach was necessary. The patient contours have also been copied on all the panels. Panel 5(a) shows the atlas after the global non rigid registration. Panel 5(b) shows the result after the local registration of the brain external contours and the ventricle. Panel 5(c) shows the final result after the local registration of the corpus callosum, the brainstem and the cerebellum. Two hierarchical layers were used: in the first layer, the registration of the external contours of the brain and of the ventricles helps to better segment the brainstem, cerebellum and corpus callosum of the second layer.

The third experience (Figure 6) consists in registering the rectum in MR slices of the prostate area, with and without constraints on the surrounding muscles contours (dark structures). For the local registration, only one level set corresponding to the rectum was used. The contours of the patient rectum have been copied on all the panels. Panel 6(a) shows the atlas after the global non rigid registration. Panel 6(b) shows the result after the level set-based registration. Panel 6(c) shows the same registration as 6(b) but using a constraint on the contours of the surrounding muscles to prevent them from being deformed by the movement of the rec- 


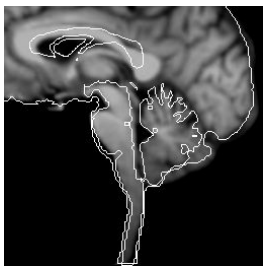

(a)

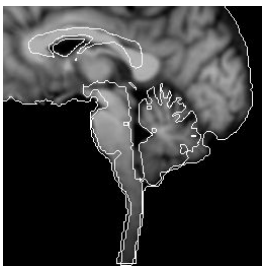

(b)

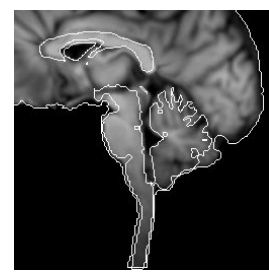

(c)
Fig. 5. Level-set based registration of a brain sagittal slice.

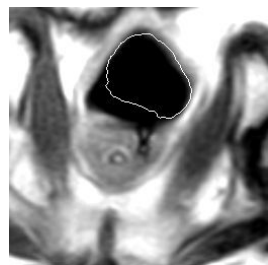

(a)

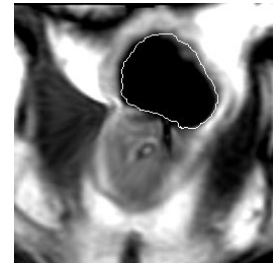

(b)

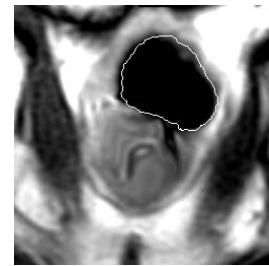

(c)
Fig. 6. Level-set based registration of a prostate axial slice.

tum. This constraint only consists in introducing the contours that can not move in both level sets used for extracting the deformation field. We can see on panel 6(c) that the left muscle is this time not attracted anymore by the rectum motion.

Three main conclusions can be drawn from these figures. First, the local registration is a good complement to an intensity-based non rigid algorithm to reach more easily and more accuracy the correct registration. Second, the hierarchical approach represents a good help to register complex structures based on objects easier to segment. Finally, we saw in the third experience that the level set tracking method permits to easily introduce constraints on contours that can not be deformed during the local registration of adjacent structures.

\section{CONCLUSION AND FUTURE WORKS}

The results presented in this paper indicate that the non rigid registration derived from the level set motion tracking represents a simple technique particularly useful to locally constrain the deformation obtained by an intensity-based atlas registration algorithm. Level set techniques permit to bring useful a priori information into the registration in addition to that already contained in the atlas. This information can concerned the contours of the patient image, the level gray distribution of interest objects, their shape or the curvature of their contours. The performance of our local registration algorithm was evaluated visually and qualitatively on 2D synthetic images and three different types of 2D medical image. Future works include testing our technique on 3D images and validating it quantitatively. We also plan to extend our local registration technique to medical images with gross abnormalities or pathologies. Its ability to propagate the deformation of some structures to the rest of the image seems to be well appropriated to this type of case. For the tumor cases, the performance of our new algorithm will be compared to those obtained with the tumor growing model previously developed in our laboratory [2]. Promising results concerning the hierarchical approach briefly introduced in this paper are presented in [13].

\section{ACKNOWLEDGEMENTS}

All this registration process has been implemented in the ITK environment [10]. This work is supported by the Swiss National Science Foundation under grant nbr 205320-101621.

\section{REFERENCES}

[1] G. K. Rohde, A. Aldroubi and B. M. Dawant, "The Adaptive Bases Algorithm for Intensity-Based Nonrigid Image Registration," IEEE Transactions on Medical Imaging, 22(11) (Nov 2003) 1470-1479.

[2] M. Bach Cuadra, C. Pollo, A. Bardera, O. Cuisenaire, J. G. Villemure and J.-P. Thiran, "Atlas-based Segmentation of Pathological MR Brain Using a Model of Lesion Growth," IEEE Transactions on Medical Imaging, 23 (10) (Oct 2004) 1301-1314.

[3] V. Duay, P.-F. D’Haese, R. Li and B. M. Dawant, "Non-Rigid Registration Algorithm With Spatially Varying Stiffness Properties," ISBI'04, (2004) 408-411.

[4] P.-F. D’Haese, V. Duay, R. Li , A. du Bois D'Aische, Th.E. Merchant, A. J. Cmelak, E. F. Donnelly, K. J. Niermann, B. Macq and B. M. Dawant, "Automatic Segmentation of Brain Structures for Radiation Therapy Planning," SPIE Medical Image Processing, (2003) 517-526.

[5] C. Baillard, P. Hellier and C. Barillot, "Cooperation between Level Set Techniques and 3D Registration for the Segmentation of Brain Structures," International Conference on Pattern Recognition (ICPR'O0), (Sep 2000) 991-994.

[6] B. C. Vemuri, J. Ye, Y. Chen and C. M. Leonard, "Image registration via level-set motion : Applications to atlas-based segmentation," IEEE Transaction on Medical Image Analysis, 7(1) (March 2003) 1-20.

[7] M. Bertalmio, G. Sapiro and G. Randall, "Morphing Active Contours," IEEE Transaction on Pattern Analysis and Machine Intelligence, 22(7) (July 2000) 733-736.

[8] D. Rueckert, L. I. Sonoda, C. Hayes, D. L. G. Hill, M. O. Leach and D. J. Hawkes, "Nonrigid registration using freeform deformations: application to breast MR images," IEEE Transactions on Medical Imaging, 18(8) (August 1999) 712721.

[9] V. Caselles, R. Kimmel and G. Sapiro, "Geodesic Active Contours," International Journal of Computer Vision, 22(1) (Feb/March 1997) 61-79.

[10] See http://www.itk.org

[11] A. Herbulot, S. Jehan-Besson, M. Barlaud and G. Aubert, "Shape gradient for image segmentation using information theory," International Conference on Acoustics, Speech, and Signal Processing (ICASSP'04), 3 (2004) 21-24.

[12] Ch. Xu and J. L. Prince, "Snakes, Shapes, and Gradient Vector Flow," IEEE Transactions on Image Processing, 7(3) (March 1998) 359-369.

[13] N. Houhou, V. Duay, A. S. Allal and J.-P. Thiran, "Medical Images Registration with a Hierarchical Atlas," EUSIPCO 2005, (Accepted). 